GLASNIK MATEMATIČKI

Vol. 41(61)(2006), $159-163$

\title{
ON INTERSECTION OF SIMPLY CONNECTED SETS IN THE PLANE
}

\author{
E. D. Tymchatyn and Vesko Valov \\ University of Saskatchewan, Canada and Nipissing University, Canada
}

\begin{abstract}
Several authors have recently attempted to show that the intersection of three simply connected subcontinua of the plane is simply connected provided it is non-empty and the intersection of each two of the continua is path connected. In this note we give a very short complete proof of this fact. We also confirm a related conjecture of Karimov and Repovš.
\end{abstract}

\section{INTRODUCTION}

A homology (resp., singular) cell is a compact metric space whose Vietoris (resp., singular) homology groups are trivial. Helly [6] proved the following result which is now known as the Topological Helly Theorem:

Theorem 1.1. Let $\mathcal{S}=\left\{S_{0}, \ldots, S_{m}\right\}, m \geq n$, be a finite family of homology cells in $\mathbb{R}^{n}$ such that the intersection of every subfamily $\mathcal{H}$ of $\mathcal{S}$ is nonempty if the cardinality $|\mathcal{H}| \leq n+1$ and it is a homology cell if $|\mathcal{H}| \leq n$. Then $\cap_{i=0}^{i=m} S_{i}$ is a homology cell.

Versions of Theorem 1.1 for singular homology have been proved by Debrunner [5]. Alexandroff and Hopf [1, p. 295] also established a simple proof of a combinatorial version of the Helly theorem.

A topological space is said to be simply connected if it is path connected and has trivial fundamental group. It is known [4] that a compact subspace of the plane is a singular cell if and only if it is simply connected.

2000 Mathematics Subject Classification. 54C55, 55M15, 54F15.

Key words and phrases. Helly theorem, plane continua, absolute retracts.

The first author was partially supported by NSERC grant NPG 0005616 .

The second author was partially supported by NSERC grant RGPIN 261914. 
In Section 2 of the paper [6] Helly proved that if $S_{i}, i=1, \ldots, 4$, are singular cells in $\mathbb{R}^{2}$ such that all intersections $S_{i_{1}} \cap S_{i_{2}} \cap S_{i_{3}}$ are singular cells, then $\cap_{i=1}^{i=4} S_{i}$ is not empty. Hence to prove the Topological Helly Theorem for singular cells in $\mathbb{R}^{2}$, it suffices to prove the following:

Proposition 1.2. Let $S_{0}, S_{1}$ and $S_{2}$ be three simply connected compacta in the plane such that the intersection of any two of them is path connected and $\cap_{i=0}^{i=2} S_{i} \neq \emptyset$. Then $\cap_{i=0}^{i=2} S_{i}$ is simply connected.

Bogatyi [2] has pointed out that no complete proof of this proposition can be found in the literature. He proved the proposition in the special case that $S_{i}$ are Peano continua. Karimov and Repovš [7], established that, with the hypotheses of Proposition 1.2, $\cap_{i=0}^{i=2} S_{i}$ is cell-like connected (i.e., every two points can be connected by a cell-like continuum). We prove Proposition 1.2 by showing that $\cap_{i=0}^{i=2} S_{i}$ is path connected. We also give an affirmative answer to a conjecture of Karimov and Repovš [7] by proving the following proposition:

Proposition 1.3. If $X$ and $Y$ are compact $A R$ 's in the plane, then so is each component of $X \cap Y$.

\section{Proof of Proposition 1.2}

Since the intersection of any family of simply connected sets in the plane has a trivial fundamental group with respect to each of its points, it suffices to show that $\cap_{i=0}^{i=2} S_{i}$ is path connected. Let $0,1 \in \cap_{i=0}^{i=2} S_{i}$ and $I \subset S_{0} \cap S_{1}$, $J \subset S_{0} \cap S_{2}$ and $K \subset S_{1} \cap S_{2}$ be arcs from 0 to 1 . Consider the components $J_{n}, n=1,2, .$. , of $J \backslash(I \cup K)$ which are not in $S_{1}$. If the family $\left\{J_{1}, J_{2}, ..\right\}$ is infinite, then $\lim _{i \rightarrow \infty} \operatorname{diam}_{i}=0$ because it is the family of components of an open set in the arc $J$. Since 0 and 1 are end-points of $J$, it follows that no $J_{i}$ separates $I \cup J \cup K$. Suppose $J_{i}$ lies in a bounded component of $\mathbb{R}^{2} \backslash(I \cup K)$. Since the locally connected continuum $I \cup K$ separates $J_{i}$ from $\infty$ in $\mathbb{R}^{2}$, some simple closed curve in $I \cup K \subset S_{1}$ does so as well [8, Chapter X, Section 61, II, Theorem 5, p. 513]. Since $S_{1}$ is simply connected, this would imply $J_{i} \subset S_{1}$, a contradiction. Thus, no $J_{i}$ lies in a bounded component of $\mathbb{R}^{2} \backslash(I \cup K)$.

We are going to construct for every $n \geq 1$ an arc $J^{n} \subset S_{0} \cap S_{2} \cap(I \cup J \cup$ $K) \backslash\left(J_{1} \cup . . \cup J_{n}\right)$ from 0 to 1 . Let $L$ be a half line irreducible from $J_{1}$ to $\infty$ in $\mathbb{R}^{2} \backslash(I \cup K)$ and $\varepsilon=\min \{d(x, y): x \in L, y \in I \cup K\}$. Then $\varepsilon>0$ because $I \cup K$ is compact and disjoint from the closed set $L$. Hence, $L$ meets only finitely many, say $\left\{J_{1}=J_{i_{1}}, . ., J_{i_{m}}\right\}$, of the sets $\left\{J_{1}, J_{2}, ..\right\}$. We may suppose $L$ meets $J_{i_{j}}$ in exactly one point for each $j \in\{1, . ., m\}$. We may also suppose that $J_{i_{j}} \cup I \cup K$ separates $J_{i_{j-1}}$ from $\infty$ in $\mathbb{R}^{2}$ for $j=2, . ., m$ if $m>1$. Give $J$ its natural linear order with initial point 0 . If $x, y \in J$ let $x y$ denote the arc in $J$ irreducible from $x$ to $y$. Now, let $x_{0}$ and $x_{1}$ be the end-points of $J_{i_{m}}$ 
with $x_{0}<x_{1}$ and let $y_{0}=\max I \cap 0 x_{0}, y_{1}=\min I \cap x_{1} 1$. Denote by $M$ the arc in $I$ irreducible from $y_{0}$ to $y_{1}$. Then $y_{0} y_{1} \cup M \subset I \cup J$ is a simple closed curve containing $J_{i_{m}}$. By the Jordan curve theorem, $y_{0} y_{1} \cup M$ bounds a disk $D$ with boundary $y_{0} y_{1} \cup M$. Let $L^{*}$ be the unbounded component of $L \backslash J_{i_{m}}$ and $D_{m} \subset D$ be the bounded component of $\mathbb{R}^{2} \backslash(I \cup J \cup K)$ whose boundary contains $J_{i_{m}}$.

Then $D_{m} \subset D_{I} \cap D_{K}$, where $D_{I}$ (resp., $\left.D_{K}\right)$ is the component of $\mathbb{R}^{2} \backslash(I \cup$ $J)$ (resp., $\mathbb{R}^{2} \backslash(J \cup K)$ ) containing $D_{m}$. Note that, as in the first paragraph of the proof, $D_{I} \subset S_{0}$ because $I \cup J \subset S_{0}$. Similarly, $D_{K} \subset S_{2}$. Thus, $D_{m} \subset S_{0} \cap S_{2}$ and $\overline{D_{m}} \subset S_{0} \cap S_{2}$ since $S_{0} \cap S_{2}$ is closed.

Moreover, $\operatorname{Fr}\left(D_{m}\right) \subset I \cup J \cup K$. It is well known [9, Theorem 2, p. 39] that each continuum contained in the union of finitely many arcs is locally connected. So $\operatorname{Fr}\left(D_{m}\right)$ is locally connected. As above, let $C \subset \operatorname{Fr}\left(D_{m}\right)$ be the simple closed curve that separates $D_{m}$ from $\infty$ in $\mathbb{R}^{2}$. Note that $J_{i_{m}} \subset C$ because $L^{*} \subset \mathbb{R}^{2} \backslash(I \cup J \cup K)$ joins $J_{i_{m}} \subset \overline{D_{m}}$ to $\infty$. Let $J^{1, m} \subset(J \cup C) \backslash J_{i_{m}} \subset$ $S_{0} \cap S_{2}$ be an arc from 0 to 1 . If $m=1$ let $J^{1}=J^{1, m}$. If $m>1$ repeat the above arguments with $J^{1, m}$ in place of $J$ and $J_{i_{m-1}}$ in place of $J_{i_{m}}$ to obtain an arc $J^{1, m-1}$ in $S_{0} \cap S_{2} \cap\left(\left(J^{1, m} \backslash J_{i_{m-1}}\right) \cup(I \cup K)\right)$ from 0 to 1 . After $m$ such steps we obtain an $\operatorname{arc} J^{1}=J^{1,1} \subset(I \cup J \cup K) \cap S_{0} \cap S_{2} \backslash\left(J_{i_{1}} \cup J_{i_{2}} \cup . . \cup J_{i_{m}}\right)$ from 0 to 1 .

Suppose we have already constructed an $\operatorname{arc} J^{n} \subset S_{0} \cap S_{2}$ in $I \cup J \cup K$ from 0 to 1 such that $J^{n} \cap\left(J_{1} \cup . . \cup J_{n}\right)=\emptyset$. If $J^{n} \cap J_{n+1}=\emptyset$, let $J^{n+1}=J^{n}$. If $J^{n} \cap J_{n+1} \neq \emptyset$, we repeat the above arguments with $J^{n}$ in place of $J$ and $J_{n+1}$ in place of $J_{1}$ to obtain an arc $J^{n+1} \subset S_{0} \cap S_{2} \cap\left(\left(J^{n} \cup I \cup K\right) \backslash J_{n+1}\right)$ from 0 to 1 . By induction, we construct a sequence of $\operatorname{arcs}\left\{J^{n}\right\}_{n=1}^{\infty}$ from 0 to 1 with

$$
J^{n+1} \subset S_{0} \cap S_{2} \cap\left((I \cup J \cup K) \backslash \bigcup_{i=1}^{n+1} J_{i}\right) .
$$

Let $J^{*}=\lim \sup J^{n}$. Then

$$
J^{*} \subset\left(S_{0} \cap S_{2}\right) \cap\left((I \cup J \cup K) \backslash \bigcup_{i=1}^{\infty} J_{i}\right) \subset S_{1}
$$

is a continuum from 0 to 1 . As above, $J^{*}$ is locally connected. So, there is an arc in $J^{*} \subset S_{0} \cap S_{1} \cap S_{2}$ from 0 to 1 .

\section{Proof of Proposition 1.3}

Let $C$ be a component of $X \cap Y$. If $K$ is the topological hull of $C$, then $K \subset X$ and $K \subset Y$ since neither $X$ nor $Y$ separates $\mathbb{R}^{2}$. So, $K=C$. By unicoherence of $\mathbb{R}^{2}$ it follows that $\operatorname{Fr}(C)$, the boundary of $C$ in $\mathbb{R}^{2}$, is connected. 
By the well-known result of Borsuk [3] (that every locally connected plane continuum not separating the plane is an $A R$ ), it remains to prove that $C$ is locally connected. Since $C$ is a continuum in the plane, it suffices to prove that $\operatorname{Fr}(C)$ is locally connected. To prove this it suffices to show that every pair of points of $\operatorname{Fr}(C)$ is separated by a finite set (see [10, p. 99]).

Since $X$ is simply connected, locally connected subcontinuum in the plane, by [10, Chapter IV], all true cyclic elements of $X$ are topological disks $D_{i}$ such that the cardinality of $D_{i} \cap D_{j}$ is at most 1 for $i \neq j$ and, if the sequence $\left\{D_{i}\right\}$ is infinite, then $\lim \operatorname{diam} D_{i}=0$. Hence, each $\operatorname{Fr}\left(D_{i}\right)$ is a simple closed curve and $\operatorname{Fr}(X)=X \backslash \bigcup \operatorname{int}\left(D_{i}\right)$ is a locally connected continuum with a particularly simple structure. Let $x$ and $y$ be distinct points in $\operatorname{Fr}(C) \subset$ $\operatorname{Fr}(X) \cup \operatorname{Fr}(Y)$. If $x$ and $y$ do not both lie in any one cyclic element of $X$, then an one point set separates $x$ and $y$ in $X$ and, hence, in $C$. Thus, we may suppose that there are cyclic elements $D$ in $X$ and $E$ in $Y$ with $x, y \in D \cap E$. Now $x$ in $\operatorname{int}(D)$ implies there is a neighborhood $W$ of $x$ in $\operatorname{Fr}(X) \cup \operatorname{Fr}(Y)$ with $\bar{W} \subset \operatorname{int}(D)$. Then a finite set $P$ separates $\operatorname{Fr}(Y) \backslash W$ from $x$ in $\operatorname{Fr}(Y)$ since $\operatorname{Fr}(Y)$ is rim-finite. Hence, $P$ separates $x$ from $\operatorname{Fr}(X) \cup \operatorname{Fr}(Y) \backslash W$. So we may suppose $x, y \in F r(D) \cap F r(E)$ (see [8, 49.V, Theorem 3, p. 244]).

Let $F$ be a two-point set in $\operatorname{Fr}(E)$ which separates $x$ and $y$ in $\operatorname{Fr}(E)$. Then $F$ separates $x$ and $y$ in $\operatorname{Fr}(Y)$ [10, IV.3.1, p. 67]. So $F \cup(D \backslash F r(Y))$ separates $x$ and $y$ in $D$. Since $D$ is hereditarily normal, a closed subset $A$ of $F \cup(D \backslash \operatorname{Fr}(Y))$ separates $x$ and $y$ in $D$. Since $D$ is unicoherent, a component $A^{\prime}$ of $A$ separates $x$ and $y$ in $D$. We can construct an arc $A^{\prime \prime} \subset D$ such that $A^{\prime \prime}$ separates $x$ and $y$ in $D$ and $A^{\prime \prime} \cap Y \subset F$. Indeed, let $N$ be an open neighbourhood of $A^{\prime} \backslash F$. So $N$ is the union of a null collection, locally finite except at points of $F$, of open balls $B_{i}$ each centered at a point of $A^{\prime} \backslash F$ and having closure in $\mathbb{R}^{2} \backslash \operatorname{Fr}(Y)$. Then the closure of $N$ and, hence, $\operatorname{Fr}(N)$ separates $x$ and $y$ in $D$. Hence, a component $G$ of $\operatorname{Fr}(N)$ separates $x$ and $y$ in $D$. As above, $G \subset \operatorname{Fr}(N) \subset \cup \operatorname{Fr}\left(B_{i}\right) \cup F$ is locally connected at each point of $G \backslash F$. Since $F$ is finite and a continuum cannot fail to be locally connected only at points of a zero dimensional set, $G$ is locally connected. Since $x$ and $y$ are on the boundary of $D$, there is an $\operatorname{arc} A^{\prime \prime}$ in $G$ which separates $x$ and $y$ in $D$.

If we also take $A^{\prime \prime}$ to be irreducible with respect to separating $x$ and $y$ in $D$ (see [8, V.49, Theorem 3, p. 244]), then $A^{\prime \prime} \cap \operatorname{Fr}(D)$ will contain just two points $c$ and $d$. As above, $A^{\prime \prime}$ separates $x$ and $y$ in $X$ because $D$ is a cyclic element of $X$. So $A^{\prime \prime} \cap(\operatorname{Fr}(X) \cup \operatorname{Fr}(Y)) \subset F \cup\{c, d\}$ separates $x$ and $y$ in $\operatorname{Fr}(C) \subset(\operatorname{Fr}(X) \cup \operatorname{Fr}(Y)) \subset X$. So, $\operatorname{Fr}(C)$ is rim-finite, hence, locally connected.

\section{ACKNOWLEDGEMENTS.}

We would like to thank the referee for his/her many valuable remarks. 
We also thank Dr. U. Karimov for pointing out an error in the first version of this note and Dr. S. Bogatyi for several fruitful discussions and comments.

\section{REFERENCES}

[1] P. Alexandroff and H. Hopf, Topologie, Chelsea, New York, 1972.

[2] S. Bogatyi, The topological Helly theorem, Fundam. Prikl. Mat. 8 (2002), 365-405 (in Russian).

[3] K. Borsuk, Sur les retracts, Fund. Math. 17 (1931), 152-170.

[4] J. W. Cannon, G. R. Conner and A. Zastrow, One-dimensional sets and planar sets are aspherical, Topology Appl. 120 (2002), 23-45.

[5] H. E. Debrunner, Helly type theorems derived from basic singular homology, Amer. Math. Monthly 77 (1970), 375-380.

[6] E. Helly, Über Systeme von abgeschlossenen Mengen mit gemeinschaftlichen Punkten, Monatsh. Math. Phys. 37 (1930), 281-302.

[7] U. Karimov and D. Repovš, On the topological Helly theorem, Topology Appl. 153 (2006), 1614-1621.

[8] K. Kuratowski, Topology, II, Academic Press, New York, 1968.

[9] N. Steenrod, Finite arc-sums, Fund. Math. 23 (1934), 38-53.

[10] G. Whyburn, Analytic Topology, Amer. Math. Soc. Colloq. Publ. 28, Providence, 1942.

[11] R. Wilder, Topology of Manifolds, Amer. Math. Soc. Colloq. Publ. 32, Providence, 1949.

E. D. Tymchatyn

Department of Mathematics and Statistics

University of Saskatchewan

McLean Hall, 106 Wiggins Road

Saskatoon, SK, S7N 5E6

Canada

E-mail: tymchat@math.usask.ca

Department of Computer Science and Mathematics

Nipissing University

100 College Drive, P.O. Box 5002

North Bay, ON, P1B 8L7

Canada

E-mail: veskov@nipissingu.ca

Received: 3.6.2004.

Revised: 20.12.2004. \& 15.6.2005. 